\title{
Consentimento Informado Normatizado pela Resolução 196/96: Conhecimento e Opinião de Pesquisadores Brasileiros
}

\author{
Knowledge and Opinion of Brazilian Researchers About Informed Consent \\ Ellen Hardy, Silvana Ferreira Bento, Maria José Duarte Osis
}

\begin{abstract}
RESUMO
Introdução: a Resolução 196/96, do Conselho Nacional de Saúde (Ministério da Saúde), apresenta as diretrizes regulamentadoras mais abrangentes acerca de pesquisas envolvendo seres humanos no Brasil, incluindo o conteúdo do termo de consentimento.

Objetivo: apresentar o conhecimento e opinião de pesquisadores brasileiros sobre o conteúdo da Resolução 196/96 do Conselho Nacional de Saúde em relação ao consentimento informado.

Sujeitos e Métodos: 46 responsáveis pela área de ginecologia em universidades, 4 diretores de centros de pesquisa e 31 pesquisadores, que participaram de um estudo sobre regulação da fecundidade nos 12 meses anteriores a setembro de 2000, completaram um questionário auto-respondido. Para a análise dos dados foi utilizado o teste de $\chi^{2}$.

Resultados: a maioria dos participantes: declarou conhecer a Resolução e que a considerava adequada, embora dificil de ser cumprida; opinou que todas as pesquisas deviam ter um termo de consentimento; sabia que o termo deve assegurar o sigilo. Significativamente mais pesquisadores do que responsáveis/diretores sabiam que o termo tinha que ser elaborado pelo pesquisador principal, em duas vias. Mais responsáveis/diretores do que pesquisadores disseram que os participantes sempre devem assinar ou colocar a impressão digital no termo e consideraram que a forma de ressarcimento das despesas decorrentes de participar no estudo deve sempre constar no documento.

Conclusões: apesar da difusão dada à Resolução 196/96, nem todos os sujeitos deste estudo a conheciam, mesmo estando ligados a uma universidade ou centro de pesquisa. A maioria foi favorável aos conteúdos exigidos pela Resolução para o consentimento informado.
\end{abstract}

PALAVRAS-CHAVE: Ética médica. Consentimento informado.

\section{Introdução}

Um conjunto de disposições internacionais tem colocado em pauta tanto os aspectos éticos da pesquisa envolvendo seres humanos como o consentimento informado. Especificamente na área da regulação da fecundidade, a Conferência Internacional de População e Desenvolvimento (ICPD),

Centro de Pesquisas Materno-Infantis de Campinas (Cemicamp)

Correspondência

Ellen Hardy

Caixa Postal 6181

13081-970 - Campinas - SP

Apoio financeiro recebido da Fundação de Amparo à Pesquisa no Estado de São Paulo (FAPESP) e do Fundo de Apoio ao Ensino e à Pesquisa (FAEP) da Universidade Estadual de Campinas (UNICAMP) realizada no Cairo, em 1994, e a Conferência Mundial sobre a Mulher - Beijing, 1995, deram grande ênfase sobre o consentimento informado como parte do exercício dos direitos sexuais e reprodutivos ${ }^{1}$.

Em relação às pesquisas, o objetivo do consentimento informado é prover a possiveis sujeitos a informação necessária para que eles possam tomar uma decisão sobre participar ou não, voluntariamente, de um estudo. Do ponto de vista ético, portanto, a exigência do consentimento informado visa a que a autonomia dos sujeitos seja respeitada, bem como se observem os princípios de beneficência e justiça ${ }^{1}$.

O governo brasileiro parece ter se interessado pela questão do consentimento informado, em relação às pesquisas envolvendo seres humanos, 
a partir de $1978^{2}$. Naquele ano, a Câmara Técnica de Medicamentos do Conselho Nacional de Saúde, do Ministério da Saúde, publicou a Resolução Normativa 1/78. Essa Resolução referia-se a aspectos éticos da experimentação terapêutica, postulando que estes deveriam estar de acordo com a Declaração de Helsinque, e afirmava que cabia ao pesquisador decidir se o consentimento dos pacientes deveria ser obtido oralmente ou por escrito.

Em 1981, a Divisão Nacional de Vigilância Sanitária de Medicamentos do Ministério da Saú$\mathrm{de}^{3}$, responsável pela regulação das drogas e medicamentos, preparou um documento-padrão, ao qual denominou de "Termo de Conhecimento de Risco", que deveria ser utilizado em ensaios com medicamentos. Porém, parece que esse documento não foi muito bem divulgado e, conseqüentemente, foi ignorado de forma ampla pelos pesquisadores do país.

Durante uma conferência realizada em 1987, o Conselho Federal de Medicina (CFM) elaborou um novo código de Ética Médica, que estabeleceu que para a realização de pesquisas com seres humanos deveria ser obtido consentimento por escrito, após esclarecer os sujeitos acerca da natureza e conseqüências da pesquisa ${ }^{4}$.

Em 1988, o Conselho Nacional de Saúde ${ }^{5}$ definiu consentimento informado como uma concordância por escrito, pela qual o sujeito da pesquisa (ou seu representante legal) aceita participar do estudo, totalmente informado sobre os procedimentos e riscos, com total independência para concordar ou não em participar, livre de qualquer forma de coerção. Quando um estudo fosse considerado de risco mínimo (por exemplo, estudos prospectivos que obtêm dados por meio de procedimentos de diagnóstico físico ou psicológico usuais ou tratamentos de rotina), o Comitê de Ética da instituição de pesquisa podia autorizar a obtenção do consentimento verbal somente. Se o estudo não fosse considerado de risco (por exemplo, aqueles nos quais não se realiza nenhuma intervenção deliberada nas variáveis fisiológicas, psicológicas ou sociais dos sujeitos, incluindo entrevistas e revisão de dados clínicos), podia ser permitido ao pesquisador proceder sem o consentimento informado.

Apesar de todas essas iniciativas, durante a Primeira Conferência Brasileira de Bioética, realizada em julho de 1996, foi afirmado que, em nosso meio, sujeitos de pesquisa raramente eram informados ou mesmo perguntados se queriam participar de um estudo ${ }^{6}$. Nesse contexto é que o Conselho Nacional de Saúde preocupou-se em revisar as normas éticas para a realização de pesquisas envolvendo seres humanos, até então estabelecidas na Resolução 1/88. Constituiu uma comissão que buscou contribuições de diversas fontes da sociedade, e acabou por publicar um documento detalhado e abrangente, a "Resolução 196/96 sobre Pesquisa Envolvendo Seres Humanos"7, atualmente em vigor.

As diretrizes definidas pela Resolução 196/ 96 visam a todas as pesquisas que envolvam seres humanos, em qualquer área do conhecimento, determinando que devem ser submetidas à apreciação de um Comitê de Ética em Pesquisa (CEP). Uma conseqüência disso foi que todas as instituições que desenvolvem pesquisas com seres humanos viram-se obrigadas a constituir um ou mais CEP. Na impossibilidade dessa medida, a Resolução 196/96 prevê que os projetos de pesquisa de uma instituição sejam apreciados pelo CEP de outra instituição, de preferência por aquele que seja indicado pela Comissão Nacional de Ética em Pesquisa (CONEP/MS).

A Resolução 196/96 também determina o que deve constar do conteúdo do Termo de Consentimento Livre e Esclarecido (TCLE), e que o mesmo deve ser avaliado pelo CEP, que decide se realmente o TCLE esclarece os potenciais sujeitos da pesquisa e permite-lhes tomar uma decisão autônoma e voluntária de participar ou não de uma pesquisa.

A divulgação da Resolução 196/96 tem sido ampla e tem mobilizado diversos setores interessados no assunto, especialmente para discutir como cumpri-la. Uma das áreas em que se tem manifestado um significativo interesse em discutir a viabilidade e a efetiva aplicação das normas estabelecidas pela Resolução 196/96 é a das pesquisas em regulação da fecundidade, há muito polemizadas em nosso meio. A própria história do movimento de mulheres no Brasil acha-se profundamente relacionada à luta em defesa da saúde das mulheres, focalizando especialmente a saúde e os direitos sexuais e reprodutivos ${ }^{8}$.

Sendo assim, o presente trabalho tem como objetivo apresentar o conhecimento e a opinião de pesquisadores brasileiros sobre o conteúdo e requisitos exigidos pela Resolução 196 para o consentimento informado.

\section{Métodos}

Realizou-se estudo descritivo com 46 responsáveis pela área de ginecologia das universidades que possuíam o curso de medicina, de acordo com levantamento do Conselho Federal de Medicina $(\mathrm{CFM})^{9}$; quatro diretores de centros que desenvolviam estudos em regulação da fecundidade, segundo o Inventário Latinoamericano de Centros 
de Investigaciones, Enseñanza y Recursos Humanos en Reproducción Humana (PLACIRH) ${ }^{10} \mathrm{e}$ 31 pesquisadores indicados pelos responsáveis / diretores, dentre os que trabalhavam com eles em suas instituições, que estavam realizando um estudo ou que haviam participado de alguma pesquisa na área de regulação da fecundidade encerrada nos últimos 12 meses anteriores a setembro de 2000.

Os dados foram obtidos por meio de questionários auto-respondidos. Para cada participante foi enviada uma carta-convite e um questionário, priorizando-se a utilização do correio eletrônico, tanto para enviar como para receber as correspondências. Quando não se identificou o endereço eletrônico, a carta convite juntamente com o questionário e um envelope pré-endereçado e selado foi enviada pela Empresa de Correios e Telégrafos. Ocasionalmente, o questionário e a carta foram enviados por fax.

Assim que os questionários foram devolvidos foi feita revisão para verificar a consistência das informações e se todas as perguntas tinham sido respondidas. Quando necessário, foi feito novo contato para esclarecer as possiveis dúvidas.

Foi preparado programa para digitar as respostas a partir dos próprios questionários, o que permitiu a verificação simultânea e interativa dos erros de digitação. Os dados foram digitados duas vezes, por pessoas diferentes. Além disso, foi feita verificação manual das freqüências das variáveis, para identificar e corrigir possiveis erros de consistência. Para avaliar as diferenças estatísticas foi utilizado o teste do $\chi^{2}{ }^{11}$.

As pessoas foram convidadas a participar voluntariamente, e esclarecidas a respeito da pesquisa por meio da carta-convite, em que se assegurou a manutenção do sigilo. $O$ fato de a pessoa responder ao questionário foi entendido como o seu consentimento. Dado o objetivo do estudo, não houve um Termo de Consentimento Livre e Esclarecido a ser assinado, porque isto sugeriria as respostas de muitas perguntas. O protocolo da pesquisa foi aprovado pela Comissão de Pesquisa do Departamento de Tocoginecologia do Centro de Assistência Integral à Saúde da Mulher (CAISM) e pelo Comitê de Ética em Pesquisa da Faculdade de Ciências Médicas da Universidade Estadual de Campinas (UNICAMP).

Para assegurar o sigilo da fonte de informação, os questionários foram identificados apenas por números. Todas as informações que permitiriam identificar a procedência dos questionários (endereço de correio eletrônico/remetente) foram removidas e destruídas/apagadas tão logo os questionários chegaram. Finalmente, após os dados terem sido digitados e o banco de dados estar com- pleto, a numeração do questionário foi refeita, alocando-se números aleatórios aos mesmos, tanto no banco de dados quanto no questionário.

Para avaliar o conhecimento e a opinião dos participantes acerca dos requisitos estabelecidos pela Resolução 196/96 para o conteúdo do consentimento informado, foram tomadas como parâmetro as determinações da Resolução. Neste trabalho são comparadas as respostas de responsáveis/diretores e pesquisadores.

\section{Resultados}

A maioria (75\%) dos participantes era do sexo masculino. A maior percentagem de mulheres $(36 \%)$ foi encontrada na categoria de pesquisadores. Em torno de três quartos dos participantes, nos dois grupos, tinha pós-graduação e quase todos eram médicos.

Quanto ao conhecimento da Resolução 196/ 96, a maioria dos responsáveis/diretores e dos pesquisadores declarou conhecê-la. Para os participantes que declararam conhecer a Resolução foi perguntado se tinham lido o documento todo ou partes dele e se a leitura foi superficial ou com atenção. A maioria disse ter lido tudo com atenção. Com relação a consultar a Resolução durante seu trabalho de pesquisa, $87 \%$ dos responsáveis/ diretores e $68 \%$ dos pesquisadores responderam que o faziam.

Não se verificou diferença estatisticamente significativa entre os grupos de participantes com relação à opinião que tinham sobre a Resolução 196/96. Mais da metade deles a considerava adequada, porém difícil de ser cumprida (67\% dos responsáveis /diretores e $75 \%$ dos pesquisadores). Pouco mais de um quinto dos dois grupos respondeu que era adequada e podia ser cumprida facilmente. Só $10 \%$ dos responsáveis/diretores e nenhum pesquisador a consideravam inadequada, dificil/impossivel de ser cumprida.

Com relação a se toda pesquisa precisa de um TCLE assinado ou só algumas, a maioria dos participantes nos dois grupos referiu que todas as pesquisas devem ter o TCLE. Para aqueles que relataram que apenas algumas pesquisas requerem o TCLE, foi apresentada uma lista com diferentes tipos de estudos para que opinassem se cada um deveria ter termo assinado ou não. Em ambos os grupos os estudos em que se testam novas drogas, os que envolvem algum tipo de risco fisico, mental e/ou emocional para o sujeito e os que retiram amostras do corpo foram os mais citados como precisando de um TCLE assinado (Tabela 1$)$. 
Tabela 1 - Opinião dos responsáveis/diretores e pesquisadores sobre quais pesquisas, envolvendo seres humanos, precisam de um TCLE assinado (em percentagem).

\begin{tabular}{|c|c|c|}
\hline & Responsáveis/Diretores \% & Pesquisadores \% \\
\hline \multicolumn{3}{|l|}{ Precisam de TCLE } \\
\hline Todas as pesquisas* & 69 & 52 \\
\hline Algumas pesquisas & 31 & 48 \\
\hline Número de entrevistados & $49^{*}$ & 31 \\
\hline Pesquisas que precisam de TCLE: Teste de drogas e tratamentos & 100 & 100 \\
\hline Riscos para saúde física dos sujeitos $\#$ & 100 & 100 \\
\hline Riscos para a saúde mental e/ou emocional dos sujeitos & 100 & 100 \\
\hline Retirar amostras de órgãos, tecidos ou fluídos do corpo* & 93 & 100 \\
\hline Só entrevistas ${ }^{@ \#}$ & 39 & 36 \\
\hline Só levantamento de dados de pastas ${ }^{\circledR \#}$ & 8 & 0 \\
\hline Outro* & 0 & 13 \\
\hline Número de entrevistados & 15 & 15 \\
\hline
\end{tabular}

Faltou a informação de: *um responsável/diretor; \# um pesquisador;

@ dois responsáveis/diretores

TCLE: Termo de consentimento livre e esclarecido

A maior parte dos participantes, nos dois grupos, concordou com os itens exigidos pela Resolução 196/96 para constarem no TCLE. Uma das exceções foi que pouco mais da metade dos participantes, nos dois grupos, considerou que as formas de indenização diante de eventuais danos decorrentes da pesquisa deviam estar no Termo.
A outra exceção, foi que a maioria $(68 \%)$ dos responsáveis/diretores considerou que a forma de ressarcimento das despesas decorrentes da participação na pesquisa deve constar sempre no TCLE, o que foi referido por menos da metade $(45 \%)$ dos pesquisadores, sendo essa diferença significativa (Tabela 2).

Tabela 2 - Porcentagem dos responsáveis/diretores e dos pesquisadores que concordaram que a inclusão dos itens exigidos pela Resolução 196/96 devem constar sempre do TCLE.

\begin{tabular}{|c|c|c|}
\hline Itens exigidos & Responsáveis/Diretores & Pesquisadores \\
\hline Garantia de sigilo\#+ & 100 & 97 \\
\hline $\begin{array}{l}\text { Liberdade de deixar o estudo em qualquer momento, sem nenhum prejuízo em seu atendimento } \\
\text { na instituição\#\# }\end{array}$ & 98 & 87 \\
\hline Garantia de esclarecimento de qualquer dúvida acerca da pesquisa, em qualquer momento* & 94 & 90 \\
\hline Liberdade de não participar do estudo, sem nenhum prejuízo em seu atendimento na instituição* & 94 & 87 \\
\hline Justificativa* & 94 & 87 \\
\hline Riscos e desconfortos possíveis ${ }^{*+}$ & 94 & 83 \\
\hline Como serão atendidos os sujeitos durante a pesquisa & 93 & 94 \\
\hline Objetivo* & 91 & 87 \\
\hline Procedimentos a serem utilizados na pesquisa*+ & 91 & 83 \\
\hline Benefícios esperados* & 89 & 84 \\
\hline Métodos alternativos existentes* & 87 & 71 \\
\hline Forma de ressarcimento das despesas decorrentes da participação na pesquisa ${ }^{\#}$ & 68 & 45 \\
\hline Formas de indenização diante de eventuais danos decorrentes da pesquisa• & 56 & 52 \\
\hline Número de entrevistados & 50 & 31 \\
\hline
\end{tabular}

Faltou a informação de: *quatro responsáveis/diretores; 'cinco responsáveis/diretores;

"seis responsáveis/diretores; 'um pesquisador; @ $p=0,046$

A Tabela 3 mostra que a maioria dos participantes dos dois grupos opinou que sempre um dos pesquisadores deve assinar o TCLE; que os pesquisadores têm direitos e obrigações/compromissos com relação aos sujeitos; que o consentimento é tão válido quando o sujeito o assina quanto 
se coloca sua impressão digital e que os sujeitos de uma pesquisa têm direitos e obrigações ou compromissos com relação aos pesquisadores. Um pouco mais da metade (63\%) dos responsáveis/diretores e quase a metade dos pesquisadores concordam que o TCLE deve ser, sempre, elaborado pelo pesquisador principal. Na opinião de quase um terço dos participantes, nos dois grupos, o consentimento, sempre, pode ser obtido por qualquer pessoa a quem o investigador principal encarregar disso.

Tabela 3 - Porcentagem dos responsáveis/diretores e dos pesquisadores que optaram por considerar corretas as afirmações citadas abaixo.

\begin{tabular}{|c|c|c|}
\hline Afirmações & Responsáveis/Diretores & Pesquisadores \\
\hline O TCLE deve ser um documento escrito* & 98 & 87 \\
\hline Os sujeitos devem assinar ou colocar sua impressão digital no TCLE*@ & 98 & 84 \\
\hline Quem está sendo convidado para ser sujeito deve receber informações sobre a pesquisa*+ & 94 & 93 \\
\hline Algum dos pesquisadores deve assinar o TCLE*+ & 93 & 97 \\
\hline Os pesquisadores têm direitos e obrigações/compromissos com relação aos sujeitos ${ }^{\bullet \varepsilon}$ & 91 & 90 \\
\hline O consentimento é tão válido quando o sujeito o assina quanto se coloca sua impressão digital ${ }^{\sharp \varepsilon}$ & 77 & 79 \\
\hline $\begin{array}{l}\text { Os sujeitos de uma pesquisa têm direitos e obrigações ou compromissos com relação aos } \\
\text { pesquisadores }{ }^{\# \varepsilon}\end{array}$ & 68 & 69 \\
\hline O TCLE deve ser elaborado pelo pesquisador principal ${ }^{\star+}$ & 63 & 47 \\
\hline $\begin{array}{l}\text { O consentimento pode ser obtido por qualquer pessoa a quem o pesquisador principal encarregar } \\
\text { disto }{ }^{\circ}\end{array}$ & 29 & 31 \\
\hline Número de entrevistados & 50 & 31 \\
\hline
\end{tabular}

Faltou a informação de:*quatro responsáveis/diretores; • cinco responsáveis/diretores; \#seis responsáveis/diretores; + $u$ m pesquisador; 'dois pesquisadores; @ p=0,036 TCLE: Termo de consentimento livre e esclarecido

Para avaliar o conhecimento acerca das exigências da Resolução 196/96 com relação ao TCLE, foram apresentadas aos participantes cinco afirmações corretas e cinco errôneas para que assinalassem se as consideravam verdadeiras ou falsas. Com respeito às afirmações corretas (as 5 primeiras), não houve diferenças significativas entre os grupos analisados, sendo que a grande maioria dos participantes classificou-as acertada- mente. Uma porcentagem significativamente menor de responsáveis/diretores do que pesquisadores classificou apropriadamente como sendo erradas duas das afirmações errôneas: o TCLE pode ser elaborado por qualquer pessoa que esteja trabalhando na pesquisa (72 e $100 \%$ respectivamente); o TCLE deve ser preparado em duas vias: uma para o pesquisador e outra para o prontuário do sujeito (13 e 47\%, respectivamente) (Tabela 4).

Tabela 4 - Porcentagem dos responsáveis/diretores e dos pesquisadores que referiram ter lido a Resolução 196/96 e que avaliaram corretamente as afirmações relacionadas às suas exigências em relação ao TCLE.

\section{Afirmações}

Deve informar acerca dos benefícios que a pesquisa poderá trazer*

Deve assegurar que o sujeito pode se recusar a participar da pesquisa ou retirar seu consentimento a qualquer momento, sem qualquer prejuízo

Deve garantir o sigilo, assegurando a privacidade dos sujeitos quanto aos dados confidenciais envolvidos na pesquisa*

Deve ser assinado (ou identificado por impressão datiloscópica) pelo sujeito ou por seu representante legal ${ }^{\bullet}$

Deve explicitar as formas de indenização diante de eventuais danos decorrentes da pesquisas`

Com respeito aos riscos basta mencionar os mais graves*

Pode ser dispensado quando a pesquisa não implicar riscos para os sujeitos

Pode ser elaborado por qualquer pessoa que esteja trabalhando na pesquisa*\#

Deve ser preparado em duas vias: uma para o pesquisador e outra para o prontuário do sujeito ${ }^{{ }^{\star}}$

Deve ser assinado também pelo pesquisador principal•

Número de entrevistados

Faltou a informação de: "três pesquisadores; ·dois pesquisadores; ${ }^{\not} p=0,018$; ${ }^{\varepsilon} p=0,009$

Responsáveis/Diretores Pesquisadores

100

100

100

94

69

84

78

72

13

13

32
90

95

95

90

80

95

90

100

47

30

22 


\section{Discussão}

Embora a Resolução 196/96 venha sendo amplamente divulgada nos meios acadêmicos e tenha mobilizado diversos setores interessados no assunto, especialmente para discutir como cumpri-la ${ }^{12}$, observou-se que, apesar de os participantes deste estudo serem chefes e/ou pesquisadores ligados a uma faculdade de medicina ou a um centro de pesquisa, nem todos estavam totalmente informados sobre a existência e o conteúdo dessa Resolução. Isto leva a refletir sobre como circulam e são assimiladas as informações no âmbito de sistemas complexos, como os de saúde e no meio acadêmico. Por exemplo, em um estudo recentemente desenvolvido no Brasil acerca da anticoncepção de emergência, evidenciou-se que, embora exista uma norma que regulamenta a provisão dessa forma de contracepção nos serviços públicos de saúde, vários profissionais entrevistados, que neles atuavam, não sabiam exatamente do que se tratava. Participantes desse estudo afirmaram que a existência da norma facilitava o trabalho dos profissionais de saúde porque oferecia amparo legal e técnico, porém, apenas a existência do documento não garantia o acesso de todos ao seu conteúdo, o que explicaria o desconhecimento observado ${ }^{13}$. Isto aponta a necessidade de que se criem mecanismos de acompanhamento para a divulgação, discussão e aplicação de normas desse tipo, o que parece ser também o caso da Resolução 196/96.

De modo geral, a maioria dos participantes mostrou concordância com as exigências da Resolução acerca do TCLE, mesmo quando declararam não conhecê-la. Isto pode ser um reflexo do processo pelo qual esse documento foi constituído, envolvendo ampla consulta a vários segmentos da sociedade brasileira, bem como fundamentado nos principais documentos internacionais que emanaram declarações e diretrizes sobre pesquisas que envolvem seres humanos ${ }^{7}$. Desta maneira, esperava-se que a Resolução refletisse a opinião da maioria dos profissionais e acadêmicos a quem ela se dirige, o que parece confirmado em nosso estudo. Entretanto, alguns pontos precisam ser considerados, na medida em que foram rejeitados pelos participantes. Um desses é a questão da indenização em caso de danos sofridos pela participação em uma pesquisa. A tendência dos participantes foi discordar da inclusão desse item no TCLE, o que já se observara em estudo anterior ${ }^{14}$. Provavelmente isso é, em parte, reflexo da polêmica que tem cercado o tema das indenizações pelos chamados "erros médicos" nos países desenvolvidos e, mais recentemente, no Brasil. De acor- do com o CFM, no país, os processos contra médicos pularam de 54 em 1988 para 315 em 1998. Entretanto, segundo um perito, muitas denúncias são infundadas. Essa tendência de processar tem suscitado até a proposta de algumas associações de médicos de fazer seguro contra isso, levantando polêmica, pois outras entidades de classe discordam ${ }^{15}$. Porém, apesar da possivel relevância desse debate, mais significativo é o fato de que, embora o pesquisador sinta-se responsável pelo sujeito, no sentido de não lhe causar dano, não dispõe de recursos no orçamento de pesquisa para prever uma possivel indenização. Vale lembrar que algumas instituições doadoras exigem que se diga o contrário do que propõe a Resolução 196/96, ou seja, que fique explicitado que não se prevêem indenizações. Este é, portanto, um impasse que precisa ser abordado com urgência pelo Conselho Nacional de Saúde, pois coloca os pesquisadores em situação delicada.

Outro aspecto a ser considerado é que uma proporção relevante dos participantes disse que não devem constar sempre do TCLE as despesas do sujeito que serão ressarcidas, decorrentes da participação no estudo. Por outro lado, essa postura pode refletir o medo dos pesquisadores de que tal ressarcimento seja confundido com pagamento pela participação, o que encobriria alguma forma de constrangimento ao possivel sujeito, eticamente inaceitável. Porém, não se pode deixar de salientar que, por mais altruístas que as pessoas sejam e desejem cooperar para o avanço científico, elas não podem arcar com o ônus econômico de sua participação numa pesquisa, que pode demandar mais deslocamentos até o hospital, clínica ou centro de pesquisa, faltas ao trabalho e necessidade de arranjos domésticos, tais como arrumar alguém para cuidar dos filhos e/ou da casa. Nesse aspecto, parece necessária uma conscientização dos pesquisadores acerca do que significa reembolsar os sujeitos pelos gastos decorrentes de sua participação numa pesquisa, de forma que isto sempre conste do TCLE. Ao mesmo tempo, sem dúvida, demanda vigilância dos Comitês de Ética no sentido de avaliarem a adequação desse reembolso, para que não se constitua em coerção para a participação dos sujeitos.

\section{ABSTRACT}

Introduction: the Resolution 196/96 of the Conselho Nacional de Saude (National Council of Health/Ministry of Health) presents the main Brazilian guidelines on research involving human subjects, including the content of written 
informed consent.

Purpose: to present the knowledge and opinion of Brazilian researchers on the contents of Resolution 196/96, specifically related to the informed consent form.

Subjects and methods: forty-six doctors responsible for the area of gynecology at Brazilian universities, four directors of research centers and 31 researchers who participated in a study related to fertility regulation during the 12 months preceding September, 2000. Subjects completed a selfreporting questionnaire. Data were analyzed by the $\chi^{2}$ test. Results: most subjects declared that they knew the Resolution 196/96 and considered it adequate, although difficult to comply with; they considered that all studies should have an informed consent form, and knew that its content should guarantee confidentiality. More researchers than those responsible for gynecology department/directors knew that the informed consent form should be prepared by the principal investigator. Significantly more responsible for gynecology department/directors than researchers declared that subjects must always sign (or put their thumb print if they do not know how to write) on the informed consent form. Subjects declared that payment of expenses resulting from participation in a study must always be explained in the informed consent form.

Conclusion: despite the wide dissemination of the Resolution 196/96, it was not known by all the researchers nor by all those responsible for gynecology departament/directors. The majority agreed with the contents required by the Resolution for the informed consent form.

KEY WORDS: Ethics Committee. Informed consent.

\section{Agradecimentos}

Os autores agradecem as instituições financiadoras, bem como a todas as pessoas que participaram desse estudo.

\section{Referências:}

1. Brenes V, Mesa A, Ortiz O, et al. El derecho al consentimiento informado: un ejercicio en construcción. Leyes, casos y procedimientos de queja en los servicios de planificación familiar en Mexico. New York: Population Council; 1998. p.56.

2. Brasil. Ministério da Saúde. Conselho Nacional de Saúde. Câmara Técnica de Medicamentos. Resolução Normativa 1/78, de 30 de agosto de 1978. Dispõe sobre o estabelecimento de uma sistemática da experimentação terapêutica, bem como de todos os itens que devem ser abrangidos nas suas diversas etapas. Diário Oficial da República Federativa do Brasil, Brasília, DF, ano CXVI; n.198, p.16746, 17 out. 1978. Seção 1-Parte 1.

3. Brasil. Ministério da Saúde. Secretaria Nacional de Vigilância Sanitária. Divisão Nacional de Vigilância Sanitária de Medicamentos (DIMED). Portaria $\mathrm{n}^{\circ}$ 16, de 27 de novembro de 1981. Dispõe sobre a instituição padronizada do Termo de Conhecimento de Risco para pesquisas com medicamentos não registrados pela DIMED, ou com indicação ainda não aprovada, ou em produtos importados ainda não analisados pela DIMED. Diário Oficial da República Federativa do Brasil, Brasília, DF, ano CXIX; n.235, p.23745, 17 out. 1978. Seção 1.

4. Conselho Federal de Medicina - CFM. Código de Ética Médica. Resolução CFM 1246/88. Rio de Janeiro: Idéia \& Produções; 1988. p. 25.

5. Brasil. Ministério da Saúde. Conselho Nacional de Saúde. Resolução nº 01/ 88 de 13 de junho de 1988. Dispõe sobre a aprovação das normas de pesquisa em saúde. Diário Oficial da República Federativa do Brasil, Brasília, DF, ano CXXVI, n.110, p.10713, 14 jun. 1988. Seção 1.

6. Normas atingirão outras áreas. JAMB J Assoc Med Bras 1996; Seção: Eventos; p.3.

7. Brasil. Ministério da Saúde. Conselho Nacional de Saúde. Normas de pesquisa envolvendo seres humanos. Res. CNS 196/96. Bioética 1996; 4 Suppl:15-25.

8. Pitanguy J. Feminist politics and reproductive rights: the case of Brazil. In: Sen G, Snow RC, editors. Power and Decision. The Social Control of Reproduction. Boston: Harvard School of Public Health; 1994.p.115.

9. Conselho Federal de Medicina. Escolas Médicas: relação de instituições de ensino superior - Curso de Medicina. [14/07/2000] 2000. Available from: URL: http:/ / cfm.org.br.

10.Placirh. Inventário latinoamericano de centros de investigaciones, enseñanza y recursos humanos en reproducción humana. México: Instituto Nacional de Ciencias Médicas y Nutrición Salvador Zubirán; 1999.p.301.

11.Armitage P. Statistical methods in medical research. 3th ed. New York: John Wiley and Sons; 1974.p.504.

12.Hardy E, Bento SF. Consentimento livre e esclarecido em pesquisas sobre regulação da fecundidade. Experiência no Brasil. Relatório Final. Apresentado à Fundação de Amparo à Pesquisa no Estado de São Paulo (FAPESP) e do Fundo de Apoio ao Ensino e à Pesquisa (FAEP) da Universidade Estadual de Campinas (UNICAMP). Campinas: Cemicamp; 2001.p.73.

13.World Health Organization. Undp/Unfpa/World Bank/World Health Organization special programme of research, development and research training in human reproduction. Final report of WHO study 96504: acceptability of emergency contraception in Latin America. Geneva: WHO; 1999.p.231.

14.Hardy E, Osis MJD, Bento SF. Informed consent and fertility regulation in Brazil. Relatório final apresentado à Organização Mundial da Saúde. Campinas: Cemicamp; 1998.p.108.

15.Globo. Época on line. Sociedade: Justiça: Médicos na retranca. [ed.147 de 26 fev. 2001]. 2001. Avaiable from: URL: http://epoca.globo.com/edic/ ed12022001/soc1ahtm

Recebido em: 7/8/2001 Aceito com modificações em: 26/11/2001 